Research Article

\title{
Novel Approaches for Getting the Solution of the Fractional Black-Scholes Equation Described by Mittag-Leffler Fractional Derivative
}

\author{
Ndolane Sene $\mathbb{B}^{1},{ }^{1}$ Babacar Sène, ${ }^{2}$ Seydou Nourou Ndiaye, ${ }^{3}$ and Awa Traoré ${ }^{4}$ \\ ${ }^{1}$ Laboratoire Lmdan, Département de Mathématiques de la Décision, Université Cheikh Anta Diop de Dakar, \\ Faculté des Sciences Economiques et Gestion, BP 5683 Dakar Fann, Senegal \\ ${ }^{2}$ Centre de Recherches Economiques Appliquées, Directeur du Laboratoire de Finances pour le Developpement (LAFIDEV), \\ Faculté des Sciences Economiques et Gestion, Université Cheikh Anta Diop de Dakar, BP 5683 Dakar Fann, Senegal \\ ${ }^{3}$ Centre de Recherches Economiques Appliquées, Laboratoire de Finances pour le Development (LAFIDEV), \\ Faculté des Sciences Economiques et Gestion, Université Cheikh Anta Diop de Dakar, BP 5683 Dakar Fann, Senegal \\ ${ }^{4}$ Centre de Recherches Economiques Appliqúees, Facultédes Sciences Economiques et Gestion, \\ Université Cheikh Anta Diop de Dakar, BP 5683 Dakar Fann, Senegal \\ Correspondence should be addressed to Ndolane Sene; ndolane.sene@ucad.edu.sn
}

Received 6 May 2020; Accepted 27 May 2020; Published 25 July 2020

Guest Editor: Qasem M. Al-Mdallal

Copyright ( $) 2020$ Ndolane Sene et al. This is an open access article distributed under the Creative Commons Attribution License, which permits unrestricted use, distribution, and reproduction in any medium, provided the original work is properly cited.

\begin{abstract}
The value of an option plays an important role in finance. In this paper, we use the Black-Scholes equation, which is described by the nonsingular fractional-order derivative, to determine the value of an option. We propose both a numerical scheme and an analytical solution. Recent studies in fractional calculus have included new fractional derivatives with exponential kernels and Mittag-Leffler kernels. These derivatives have been found to be applicable in many real-world problems. As fractional derivatives without nonsingular kernels, we use a Caputo-Fabrizio fractional derivative and a Mittag-Leffler fractional derivative. Furthermore, we use the Adams-Bashforth numerical scheme and fractional integration to obtain the numerical scheme and the analytical solution, and we provide graphical representations to illustrate these methods. The graphical representations prove that the Adams-Bashforth approach is helpful in getting the approximate solution for the fractional Black-Scholes equation. Finally, we investigate the volatility of the proposed model and discuss the use of the model in finance. We mainly notice in our results that the fractional-order derivative plays a regulator role in the diffusion process of the Black-Scholes equation.
\end{abstract}

\section{Introduction}

There are many mathematical models [1-3] used in finance to predict the values of cost, revenue, and options. In this paper, we address the application of fractional calculus in economics and finance. Fractional derivatives occupy an important place in fractional calculus, so this paper investigates the use of fractional derivatives for modeling financial and economic models. The Black-Scholes model is an important tool used in finance to predict the value of an option [2]. There are many styles of options: European options $[3,4]$, American options [5, 6], and Asian options. The pricing of options is a subject that has been very intensely debated in economics. So far, the economic and financial literature continues to take an interest in this subject. Today, the questions arise concerning alternative methods for pricing European options, which are derivatives that can only be exercised at maturity. The literature is also interested in the valuation of American options (there is not yet a consensus model). American options are derivatives that can be exercised at any time. The methodology proposed in this paper, therefore, makes it possible to price a European option. It could be extended to American and Asian options and knock-in and knock-out barrier options. Another possible application is the determination of the default risk of bonds listed on the financial market. The model could 
possibly be applied to determine the unknowns of the Merton-Black-Scholes-based model. Note that predicting the exact value of the American option is an ongoing problem that is generally not common knowledge. For European options, the model proposed by Black and Scholes [2] has an exact analytical solution and a numerical scheme and that analytical solution uses the normal distribution. The use of the normal distribution in the formula for an option is not always suitable; thus, our work proposes an analytical solution to this problem using recursive approximation that avoids the need for a normal distribution in the formula for the value of a European option.

Some studies in the context of fractional calculus have investigated the use of the Black and Scholes equation for European options. Much of this research has involved the use of the Caputo-Liouville and the Riemann-Liouville derivatives. In [4], Fall et al. offered a new work on fractional Black-Scholes equations described by the generalized fractional derivative. In [4], the authors present a new procedure to obtain the analytical solutions of the fractional Black-Scholes model, which is called the homotopy perturbation method. Sawangston et al. proposed an analytical solution for the fractional Black-Scholes equation with the Caputo-Liouville derivative in [7]. The research in [8] offered an analytical solution for the fractional Black-Scholes described by the conformable derivative. For more numerical schemes proposed for the Black-Scholes equation, see $[1,6,9-13]$. For other numerical procedures in other differential equations, the readers can refer to [14-20].

Modeling the physical phenomena using the fractionalorder derivative has many advantages. First of all, it permits us to study the differential models with arbitrary nonintegerorder derivatives. Second, it permits us to take into account the memory effect; that is, the next behavior of the dynamic is explained by the past behavior of the dynamic. As we will observe in this paper, the fractional-order derivative can play a regulator role in differential dynamics. In this paper, we investigate and introduce the fractional Black-Scholes equation described by a new fractional-order derivative: the Mittag-Leffler fractional derivative [21]. We prove this can be used in finance to evaluate the value of an option. Note that the new model uses the Atangana-Baleanu derivative and takes into account the integer-order time derivative. We propose a numerical scheme of the introduced model using the Adams-Bashforth method and an analytical solution using the recursive procedure proposed by Liao in [22]. Finally, we use the analytical solution to analyse the volatility of the option to a change in the price of the underlying security.

This manuscript is structured as follows: In Section 2, we define the fractional derivatives with Mittag-Leffler kernels. In Section 3, we introduce and discuss the fractional Black-Scholes equation described by the Mittag-Leffler fractional derivative. In Section 4, we prove the existence and uniqueness of our introduced model. In Section 4, we describe the Adams-Bashforth method. In Section 5, we propose the numerical discretization of the fractional Black-Scholes equation. In Section 6, we propose an analytical solution for the Black-Scholes equation described by the Atangana-Baleanu fractional derivative. In Section 7, we analyse the volatility of the fractional Black-Scholes equation, and in Section 8, we present graphical representations and a discussion. Section 9, includes our final remarks and conclusions.

\section{Fractional Operators without Singular Kernels}

In this section, we recall the definitions of fractional derivatives with nonsingular kernels. Fractional calculus began with the Riemann-Liouville fractional derivative and the Caputo-Liouville fractional derivative; for definitions, refer to [23-25]. These two derivatives were proposed in response to Leibniz's question in 1695. Many other fractional derivatives were introduced in the literature after this date and have been applied in physics, mechanics, and mathematical modeling [25-31]. Some properties were lost in modeling real-world phenomena, specifically the physical aspects. Recently, Caputo and Fabrizio proposed a new fractional derivative with an exponential kernel [32] and gave it an associated fractional integral. The main advantage of this fractional derivative is its lack of singularity, and it can be used in modeling many physical phenomena [26, 33]. The definitions are given in the following.

Definition 1 (see [32]). The Caputo-Fabrizio fractional derivative of the function $u: \mathbb{R} \times[0,+\infty[\longrightarrow \mathbb{R}$ of order $\alpha$ is defined in the form

$$
D_{\alpha}^{\mathrm{CF}} u(y, t)=\frac{M(\alpha)}{1-\alpha} \int_{0}^{t} u^{\prime}(y(s), s) \exp \left(-\frac{\alpha}{1-\alpha}(t-s)\right) \mathrm{d} s,
$$

where $t>0$ and the order $\alpha \in(0,1)$, with the normalization term satisfying $M(0)=M(1)=1$.

Definition 2 (see [32]). The Caputo-Fabrizio integral for a given function $u: \mathbb{R} \times[0,+\infty[\longrightarrow \mathbb{R}$, of order $\alpha \in(0,1]$, is defined in the form

$$
\begin{aligned}
I_{\alpha}^{\mathrm{CF}} u(y, t)= & \frac{2(1-\alpha)}{(2-\alpha) M(\alpha)} u(y, t) \\
& +\frac{2 \alpha}{(2-\alpha) M(\alpha)} \int_{0}^{t} u(y(s), s) \mathrm{d} s,
\end{aligned}
$$

for all $t>0$ and the order $\alpha \in(0,1)$, with the normalization term satisfying $M(0)=M(1)=1$.

The exponential form is a particular case of the MittagLeffler function. Motivated by the fact that the Cauchy problem with the Caputo-Fabrizio derivative generates a solution with an exponential function, Atangana and Baleanu proposed another fractional derivative with a Mittag-Leffler kernel in 2016 [21]. In other words, we can translate the Cauchy equation using Caputo-Fabrizio as the first-order equation with an integer-order derivative.

Definition 3 (see [21]). The Atangana-Baleanu-Caputo derivative for a function $u: \mathbb{R} \times[0,+\infty[\longrightarrow \mathbb{R}$, of order $\alpha \in(0,1]$, is defined in the form 


$$
D_{\alpha}^{\mathrm{ABC}} u(y, t)=\frac{B(\alpha)}{1-\alpha} \int_{0}^{t} u^{\prime}(y(s), s) E_{\alpha}\left(-\frac{\alpha}{1-\alpha}(t-s)^{\alpha}\right) \mathrm{d} s
$$

for all $t>0$, where the function $\Gamma(\ldots)$ is the Euler gamma function and $E_{\alpha}(\cdot)$ denotes the Mittag-Leffler function, with the normalization term satisfying $B(0)=B(1)=1$.

Definition 4 (see [21]). The Atangana-Baleanu integral for a given function $u: \mathbb{R} \times[0,+\infty[\longrightarrow \mathbb{R}$, of order $\alpha \in(0,1]$, is defined as the form

$$
\begin{aligned}
I_{\alpha}^{\mathrm{AB}} u(y, t)= & \frac{1-\alpha}{B(\alpha)} u(y, t) \\
& +\frac{\alpha}{B(\alpha)} \frac{1}{\Gamma(\alpha)} \int_{0}^{t}(t-s)^{\alpha-1} u(y(s), s) \mathrm{d} s
\end{aligned}
$$

for all $t>0$, where the function $\Gamma(\ldots)$ is the Euler gamma function and $E_{\alpha}(\cdot)$ denotes the Mittag-Leffler function, with the normalization term satisfying $B(0)=B(1)=1$.

We were motivated to consider these two fractional derivatives because of their successful application in modeling real-life phenomena. In this paper, we apply the Atangana-Baleanu fractional derivative in modeling the value of options and investigate the fractional Black-Scholes equation described by the Atangana-Baleanu-Caputo fractional derivative.

\section{Black-Scholes in the Context of Mittag- Leffler Fractional Derivative}

In this section, we introduce the Black-Scholes equation in the context of the Atangana-Baleanu fractional derivative. We begin by recalling the classical model proposed by Black and Scholes [2]. Let the asset price be $S$ at time, the constant volatility of an underlining asset be represented by the parameter $\sigma$, and $\mu$ be the expected rate of return. Myron and Fischer stipulated that the stock price follows a Brownian motion denoted by the parameter $w$; thus, we have the following:

$$
\mathrm{d} S=\mu \mathrm{S} d t+\sigma S \mathrm{~d} w
$$

Note that equation (5) describes the asset price $S$ as Brownian motion and represents a particular case of Ito's lemma. There are two types of derivations in the literature for pricing options: in this paper, we use Fisher and Myron's derivation. Black and Scholes expressed the value of the portfolio denoted by $P$ in the following form:

$$
\mathrm{d} P=\mathrm{d} V-\frac{\partial V}{\partial S} \mathrm{~d} S,
$$

where $V$ represents the value of an option. We have decomposed the portfolio into the value of the option and the asset price. $\partial V / \partial S \mathrm{~d} S$ denotes the asset price obtained per year. Using Ito's lemma, equation (6) can be represented in the following form:

$$
\begin{aligned}
\mathrm{d} P= & {\left[\frac{\partial V}{\partial \tau}+\mu S \frac{\partial V}{\partial S}+\frac{\sigma^{2}}{2} S^{2} \frac{\partial V^{2}}{\partial S^{2}}-\frac{\partial V}{\partial S} S \mu\right] \mathrm{d} t } \\
& +\left[\sigma S \frac{\partial V}{\partial S}-\sigma \frac{\partial V}{\partial S} S\right] \mathrm{d} w .
\end{aligned}
$$

Taking into account the interest rate $r$ into the value of the portfolio, we express equation (6) in the following form:

$$
\mathrm{d} P=r V \mathrm{~d} t-r \frac{\partial V}{\partial S} \mathrm{~S} \mathrm{~d} t
$$

Combining equations (7) and (8), the differential equation which calculates the value of a European option is given by the following equation:

$$
\frac{\partial V}{\partial t}+\frac{\sigma^{2}}{2} S^{2} \frac{\partial^{2} V}{\partial S^{2}}+r S \frac{\partial V}{\partial S}-r V=0
$$

We made the following assumptions related to equation (9): it considers a European option, the risk-less interest rate $r$ is constant, and there are no transaction costs, and we authorize the possibility to buy and to sell any number of stocks with no restriction to short selling at the last moment. The boundary conditions for equation (9) are defined as $V(0, t)=0, V(S, T) \approx S$ as $S \longrightarrow \infty$, and the terminal condition is given by

$$
V(S, T)=\max (S-E, 0)
$$

where the parameter $E$ denotes the strike price of the underlying stock and $T$ represents the expiration time. For the European option, we have the possibility to buy and to exercise the option with no obligation at time $T$. That means we can sell the risky asset to a seller at a strike price $E$. Equation (10) can be explained as follows: we exercise the option at time $T$ when the condition $E<S$ is held. That is, $V(S, T)=\max (S-E, 0)=$ $S-E$; in other words, the buyer receives the playoff $S-E$. The benefit is in selling the asset to the seller of the contract rather than on the financial market. When the condition $S<E$ is held, the contract is not good for the buyer, and the buyer can sell the risky asset for a larger price on the financial market.

The use of equation (9) for the analytical solution or the numerical scheme is not trivial, but equation (9) is a diffusion equation and can be rewritten more simply. We use the following changes to the variables described by the relationships:

$$
\begin{gathered}
S=E e^{x}, \\
t=T-\frac{2 \tau}{\sigma^{2}}, \\
V=E u(x, t) .
\end{gathered}
$$
by

From which it follows the classical Euler equation given

$$
\frac{\partial u}{\partial \tau}=\frac{\partial^{2} u}{\partial x^{2}}+(k-1) \frac{\partial u}{\partial x}-k u
$$

with the initial boundary condition defined by 


$$
u(x, 0)=\max \left(e^{x}-1,0\right)
$$

where $k=2 r / \sigma^{2}$ denotes the balance between the free interest rate and the volatility of the stocks. The difference between equation (9) and equation (12) shows the transition between the finance model and the physical model. Note that it was provided in fractional calculus; many models described by the classical derivative as in equation (9) cannot describe the real behavior of the modeled phenomena. It has been proved that many real-world problems follow fractional phenomena. Equation (12) is a diffusion equation, and there are many diffusion processes in physics, such as subdiffusion, superdiffusion, ballistic diffusion, and hyperdiffusion, which equation (12) does not take into account. This problem requires the introduction of a fractional derivative that takes into account all types of diffusion processes. In this paper, we replace the ordinary time derivative with the fractional-order time derivative, and we describe the fractional differential equation which we consider using the following fractional differential equation:

$$
D_{\alpha}^{\mathrm{ABC}} u=\frac{\partial^{2} u}{\partial x^{2}}+(k-1) \frac{\partial u}{\partial x}-k u
$$

with the initial boundary condition defined by

$$
u(x, 0)=\max \left(e^{x}-1,0\right)
$$

In the next section, we try to prove our new model is well defined, admit a unique solution, and use numerical and analytical methods to approach it. For the readers and more understanding of the paper, we summarize the description of the parameters used in this paper in Table 1.

\section{Adams-Bashforth Numerical Approach}

In this section, we describe the procedure of discretization used in this paper. The method is called the Adams-Bashforth numerical scheme and was introduced in fractional calculus by Atangana in [34]. Adams-Bashforth is a useful method that involves the following fundamental theorem [34].

Theorem 1 (see [34]). The solution of the fractional differential equation described by $D_{\alpha}^{A B C} v=f(v, t)$ with initial boundary condition $v(0)$ satisfies the following relationship:

$$
v(t)-v(0)=\frac{1-\alpha}{B(\alpha)} f(v, t)+\frac{\alpha}{B(\alpha)} \int_{0}^{t}(t-s)^{\alpha-1} f(v, s) \mathrm{d} s .
$$

The approximation of the function $f$ uses a Lagrange polynomial, which is the main novelty of Atangana's proposed numerical approximation. The following relationship describes the Lagrange polynomial:

$$
p(t)=\frac{t-t_{n-1}}{t_{n}-t_{n-1}} f\left(v_{n}, t_{n}\right)+\frac{t-t_{n-1}}{t_{n-1}-t_{n}} f\left(v_{n-1}, t_{n-1}\right) .
$$

Using equation (17), the discretized approximation of equation (16) at time $t_{n+1}$ and $t_{n}$, considering $h=t_{n}-t_{n-1}$ as described in [34], is obtained by the following equation:

$$
v\left(t_{n+1}\right)-v\left(t_{n}\right)=\theta(\alpha, 1)+\theta(\alpha, 2),
$$

where the function $\theta(\alpha, 1)$ is given by the expression described in the following equation:

$$
\theta(\alpha, 1)=f\left(v_{n}, t_{n}\right)\left\{\frac{1-\alpha}{B(\alpha)}+\frac{\alpha}{B(\alpha) \Gamma(\alpha) h}\left(\frac{2 h t_{n+1}^{\alpha+1}}{\alpha}-\frac{t_{n+1}^{\alpha+1}}{\alpha+1}\right)-\frac{\alpha}{B(\alpha) \Gamma(\alpha) h}\left(\frac{h t_{n}^{\alpha}}{\alpha}-\frac{t^{\alpha+1}}{\alpha+1}\right)\right\}
$$

and where the function $\theta(\alpha, 2)$ is given by the expression described in the following equation:

$$
\theta(\alpha, 2)=f\left(v_{n-1}, t_{n-1}\right)\left\{\frac{\alpha-1}{B(\alpha)}-\frac{\alpha}{B(\alpha) \Gamma(\alpha) h}\left(\frac{h t_{n+1}^{\alpha}}{\alpha}-\frac{t_{n+1}^{\alpha+1}}{1+\alpha}+\frac{t^{\alpha+1}}{B(\alpha) \Gamma(\alpha) h}\right)\right\} .
$$

The above discretization proposed by Atangana in [34] is very useful in numerical solutions for fractional differential equations described by certain fractional derivatives, such as the Caputo fractional derivative and the fractional derivative with the Mittag-Leffler kernel. The application of the Adams-Bashforth scheme uses the discretization of the terms $f\left(v_{n}, t_{n}\right)$ and $f\left(v_{n-1}, t_{n-1}\right)$. We can do the standard discretization procedures to discretize them. Here, we use the central difference schemes for the second-order space derivative and numerical approximation of the first-order space derivative. Before moving on, we look at the stability of the method used and see that stability is obtained when the function $f$ is Lipschitzian. Using equations (19) and (20), the following relationship is obtained:

$$
\begin{aligned}
v\left(t_{n+1}\right)-v\left(t_{n}\right)= & \frac{1-\alpha}{B(\alpha)}\left[f\left(v_{n}, t_{n}\right)-f\left(v_{n-1}, t_{n-1}\right)\right] \\
& +\Omega(\alpha, 1)-\Omega(\alpha, 2),
\end{aligned}
$$

where the function $\Omega(\alpha, 1)$ is given by the relationship

$$
\Omega(\alpha, 1)=\frac{\alpha}{B(\alpha)} \int_{0}^{t_{n+1}}\left(t_{n+1}-s\right)^{\alpha-1} f(v, s) \mathrm{d} s,
$$


TABle 1: Parameters of the Black-Scholes equation.

\begin{tabular}{lc}
\hline Parameters & Description of the parameters. \\
\hline$V$ and $T$ & The value of an option and the expiration time, respectively. \\
$\sigma$ & The volatility of the underlining stock. \\
$k$ & The balance between the free interest rate and the volatility of the stock. \\
$r$ & The risk-less interest rate.
\end{tabular}

and the function $\Omega(\alpha, 2)$ is given by the relationship

$$
\Omega(\alpha, 2)=\frac{\alpha}{B(\alpha)} \int_{0}^{t_{n}}\left(t_{n}-s\right)^{\alpha-1} f(v, s) \mathrm{d} s .
$$

We will find a threshold for the function $\Omega(\alpha, 1)-\Omega(\alpha, 2)$ by applying the Euclidean norm:

$$
\begin{aligned}
\|\Omega(\alpha, 1)-\Omega(\alpha, 2)\| & \leq\left\|\frac{\alpha}{B(\alpha)} \int_{0}^{t_{n+1}}\left(t_{n+1}-s\right)^{\alpha-1} f(v, s) \mathrm{d} s-\frac{\alpha}{B(\alpha)} \int_{0}^{t_{n}}\left(t_{n}-s\right)^{\alpha-1} f(v, s) \mathrm{d} s\right\| \\
& \leq\left\|\frac{\alpha}{B(\alpha)} \int_{0}^{t_{n+1}}\left(t_{n+1}-s\right)^{\alpha-1} f(v, s) \mathrm{d} s\right\|+\left\|\frac{\alpha}{B(\alpha)} \int_{0}^{t_{n}}\left(t_{n}-s\right)^{\alpha-1} f(v, s) \mathrm{d} s\right\| \\
& \leq \frac{\alpha}{B(\alpha)} \int_{0}^{t_{n+1}}\left(t_{n+1}-s\right)^{\alpha-1}\|f(v, s)\| \mathrm{d} s+\frac{\alpha}{B(\alpha)} \int_{0}^{t_{n}}\left(t_{n}-s\right)^{\alpha-1}\|f(v, s)\| \mathrm{d} s \\
& \leq \frac{\alpha M}{B(\alpha)} \int_{0}^{t_{n+1}}\left(t_{n+1}-s\right)^{\alpha-1} \mathrm{~d} s+\frac{\alpha M}{B(\alpha)} \int_{0}^{t_{n}}\left(t_{n}-s\right)^{\alpha-1} \mathrm{~d} s \\
& \leq \frac{\alpha M}{B(\alpha) \Gamma(\alpha+1)}\left[t_{n+1}^{\alpha}+t_{n}^{\alpha}\right]=\frac{\alpha M h^{\alpha}}{B(\alpha) \Gamma(\alpha+1)}\left[(n+1)^{\alpha}+(n)^{\alpha}\right] .
\end{aligned}
$$

Using the assumption $t_{n}=n h$, we get the following relationship:

$$
\|\Omega(\alpha, 1)-\Omega(\alpha, 2)\| \leq \frac{\alpha M h^{\alpha}}{B(\alpha) \Gamma(\alpha+1)}\left[(n+1)^{\alpha}+(n)^{\alpha}\right] .
$$

Applying the norm to both sides of equation (21), we obtain the following relationships:

$$
\begin{aligned}
\left\|v\left(t_{n+1}\right)-v\left(t_{n}\right)\right\| \leq & \frac{1-\alpha}{B(\alpha)}\left\|\left[f\left(v_{n}, t_{n}\right)-f\left(v_{n-1}, t_{n-1}\right)\right]\right\| \\
& +\|\Omega(\alpha, 1)-\Omega(\alpha, 2)\|, \\
\leq & \frac{1-\alpha}{B(\alpha)}\left\|\left[f\left(v_{n}, t_{n}\right)-f\left(v_{n-1}, t_{n-1}\right)\right]\right\| \\
& +\frac{\alpha M h^{\alpha}}{B(\alpha) \Gamma(\alpha+1)}\left[(n+1)^{\alpha}+(n)^{\alpha}\right] .
\end{aligned}
$$

From equation (26), it can be seen that when the function $f$ is locally Lipschitz and $h$ converges to zero, we obtain the following relationship:

$$
\left\|v\left(t_{n+1}\right)-v\left(t_{n}\right)\right\| \longrightarrow 0 .
$$

We can conclude the Adams-Bashforth numerical scheme is unconditionally stable. In the next section, we apply the Adams-Bashforth numerical scheme to the numerical approximation of the fractional Black-Scholes equation described by the fractional derivative with MittagLeffler.

\section{Numerical Approach for Fractional Black-Scholes Equation}

In this section, we describe the Adams-Bashforth numerical scheme for the fractional Black-Scholes equation represented by the Atangana-Baleanu fractional derivative. Let us begin the numerical approximation of the fractional Black-Scholes equation. Let $t_{n}=n h$. The Adams-Bashforth numerical scheme for the fractional Black-Scholes equation described by the Atangana-Baleanu fractional derivative takes the following form:

$$
u\left(t_{n+1}\right)-u\left(t_{n}\right)=\theta(\alpha, 1)-\theta(\alpha, 2),
$$

where the function $\theta(\alpha, 1)$ is given by the expression described in the following equation: 


$$
\theta(\alpha, 1)=f\left(u_{n}, t_{n}\right)\left\{\frac{1-\alpha}{B(\alpha)}-\frac{\alpha}{B(\alpha) \Gamma(\alpha)} h^{\alpha}\left[\frac{2(n+1)^{\alpha}}{\alpha}-\frac{(n+1)^{\alpha+1}}{\alpha+1}\right]-\frac{\alpha}{B(\alpha) \Gamma(\alpha)} h^{\alpha}\left[\frac{(n)^{\alpha}}{\alpha}-\frac{(n)^{\alpha+1}}{\alpha+1}\right]\right\}
$$

and where the function $\theta(\alpha, 2)$ is given by the expression described in the following equation:

$$
\theta(\alpha, 2)=f\left(u_{n-1}, t_{n-1}\right)\left\{\frac{1-\alpha}{B(\alpha)}-\frac{\alpha}{B(\alpha) \Gamma(\alpha)} h^{\alpha}\left[\frac{(n+1)^{\alpha}}{\alpha}-\frac{(n+1)^{\alpha+1}}{\alpha+1}+\frac{n^{\alpha+1}}{B(\alpha) \Gamma(\alpha) h}\right]\right\}
$$

The next step consists of finding the discretization of the functions $f\left(v_{n}, t_{n}\right)$ and $f\left(v_{n-1}, t_{n-1}\right)$. In the Black-Scholes equation, the function $f$ is given by

$$
f(u, t)=\frac{\partial^{2} u}{\partial x^{2}}+(k-1) \frac{\partial u}{\partial x}-k u \text {. }
$$

Using the central difference approximation for the second-order derivative with respect to the space coordinate and the numerical approximation for the space derivative, we obtain the following discretization at the points $u_{n}$ and $t_{n}$ for the function $f$ :

$$
\begin{aligned}
f\left(u_{n}, t_{n}\right)= & \frac{u_{j+1}^{n}-2 u_{j}^{n}+u_{j-1}^{n}}{\Delta x^{2}}+(k-1) \frac{u_{j+1}^{n}-u_{j-1}^{n}}{2 \Delta x} \\
& -k u_{j}^{n}+O(\Delta x) .
\end{aligned}
$$

Using the central difference approximation again for the second-order derivative with respect to the space coordinate and the numerical approximation for the space derivative, we obtain the following discretization at the points $u_{n-1}$ and $t_{n-1}$ for the function $f$ :

$$
\begin{aligned}
f\left(u_{n-1}, t_{n-1}\right)= & \frac{u_{j+1}^{n-1}-2 u_{j}^{n-1}+u_{j-1}^{n-1}}{\Delta x^{2}}+(k-1) \frac{u_{j+1}^{n-1}-u_{j-1}^{n-1}}{2 \Delta x} \\
& -k u_{j}^{n-1}+O(\Delta x)
\end{aligned}
$$

Numerical discretization using the Adams-Bashforth method for the Black-Scholes equation is obtained by combining equations (28)-(33). That is,

$$
u_{j}^{n+1}=u_{j}^{n}+\theta(\alpha, 1)-\theta(\alpha, 2) \text {. }
$$

For the computation of our numerical schemes, we make some changes to the variables, such that the terms depending on $n, h$, and $\alpha$ are constants. Thus, we rewrite $\theta(\alpha, 1)$ and $\theta(\alpha, 2)$, respectively, as in the following relationships:

$$
\begin{aligned}
& H(n, h, \alpha, 1)=\left\{\frac{1-\alpha}{B(\alpha)}-\frac{\alpha}{B(\alpha) \Gamma(\alpha)} h^{\alpha}\left[\frac{2(n+1)^{\alpha}}{\alpha}-\frac{(n+1)^{\alpha+1}}{\alpha+1}\right]-\frac{\alpha}{B(\alpha) \Gamma(\alpha)} h^{\alpha}\left[\frac{(n)^{\alpha}}{\alpha}-\frac{(n)^{\alpha+1}}{\alpha+1}\right]\right\}, \\
& H(n, h, \alpha, 2)=\left\{\frac{1-\alpha}{B(\alpha)}-\frac{\alpha}{B(\alpha) \Gamma(\alpha)} h^{\alpha}\left[\frac{(n+1)^{\alpha}}{\alpha}-\frac{(n+1)^{\alpha+1}}{\alpha+1}+\frac{n^{\alpha+1}}{B(\alpha) \Gamma(\alpha) h}\right]\right\} .
\end{aligned}
$$

Finally, the numerical scheme using the Adams-Bashforth method for the fractional Black-Scholes equation is given by

$$
\begin{aligned}
u_{j}^{n+1}= & u_{j}^{n}+H(n, h, \alpha, 1)\left[\frac{u_{j+1}^{n}-2 u_{j}^{n}+u_{j-1}^{n}}{\Delta x^{2}}+(k-1) \frac{u_{j+1}^{n}-u_{j-1}^{n}}{2 \Delta x}-k u_{j}^{n}\right] \\
& -H(n, h, \alpha, 2)\left[\frac{u_{j+1}^{n-1}-2 u_{j}^{n-1}+u_{j-1}^{n-1}}{\Delta x^{2}}+(k-1) \frac{u_{j+1}^{n-1}-u_{j-1}^{n-1}}{2 \Delta x}-k u_{j}^{n-1}\right] . \\
& u_{0}^{n}(x)=\max \left(e^{x}-1,0\right) .
\end{aligned}
$$

To complete the numerical discretization given in

equation (37), we recall the discretized form of the initial boundary condition described by the following expression: 


\section{Analytical Solution for Fractional Black-Scholes Equation}

In this section, we use the fractional integrator to propose the analytical solution of the fractional Black-Scholes equation described by the Atangana-Baleanu fractional derivative. The method is described in the following theorem.

Theorem 2. The solution of the fractional differential equation described by $D_{\alpha}^{A B C} v=f(v, \tau)$ with initial boundary condition $v(0)$ satisfies the following relationship:

$$
v_{n+1}(\tau)-v_{n+1}(0)=\frac{1-\alpha}{B(\alpha)} f\left(v_{n}, \tau\right)+\frac{\alpha}{B(\alpha)} \int_{0}^{\tau} f\left(s, v_{n}\right) \mathrm{d} s,
$$

where $n=0,1,2, \ldots$ Furthermore, the solution is given by

$$
v(x, \tau)=v_{0}(0)+v_{1}(\tau)+v_{2}(\tau)+\ldots
$$

Consider the fractional Black-Scholes equation (14). Using Theorem 2, with assumption $u_{1}(x, 0)=0$, we have the following solution, which is the first step:

$$
\begin{aligned}
u_{1}(x, \tau) & =u_{1}(x, 0)+\frac{1-\alpha}{B(\alpha)} k\left(\max \left(e^{x}, 0\right)-\max \left(e^{x}-1,0\right)\right)+\frac{k\left(\max \left(e^{x}, 0\right)-\max \left(e^{x}-1,0\right)\right) \tau^{\alpha}}{B(\alpha) \Gamma(1+\alpha)} \\
& =\frac{1-\alpha}{B(\alpha)} k\left(\max \left(e^{x}, 0\right)-\max \left(e^{x}-1,0\right)\right)+\frac{\alpha k\left(\max \left(e^{x}, 0\right)-\max \left(e^{x}-1,0\right)\right) \tau^{\alpha}}{B(\alpha) \Gamma(1+\alpha)} \\
& =\left[k\left(\max \left(e^{x}, 0\right)-\max \left(e^{x}-1,0\right)\right)\right]\left\{\frac{1-\alpha}{B(\alpha)}+\frac{\alpha \tau^{\alpha}}{B(\alpha) \Gamma(1+\alpha)}\right\} .
\end{aligned}
$$

Under assumption $u_{2}(x, 0)=0$, again using equation (39), the second step gives the following solution:

$$
\begin{aligned}
& u_{2}(x, \tau)= {\left[k^{2}\left(\max \left(e^{x}, 0\right)-\max \left(e^{x}-1,0\right)\right)\right](1-\alpha)\left\{\frac{1-\alpha}{B(\alpha)}+\frac{\alpha \tau^{\alpha}}{B(\alpha)} \Gamma(1+\alpha)\right\} } \\
&-\left[k^{2}\left(\max \left(e^{x}, 0\right)-\max \left(e^{x}-1,0\right)\right)\right](\alpha)\left\{\frac{(1-\alpha) \tau^{\alpha}}{B(\alpha) \Gamma(1+\alpha)}+\frac{\alpha \tau^{2 \alpha}}{B(\alpha) \Gamma(1+2 \alpha)}\right\} . \\
& u(x, \tau)=\max \left(e^{x}, 0\right)\left(1-e^{-k \tau}\right)+\max \left(e^{x}-1,0\right) e^{-k \tau}
\end{aligned}
$$

We adopt the procedure described in Theorem 2 for the rest of the steps. The following expression gives the approximate solution of the fractional Black-Scholes equation in the context of the Mittag-Leffler fractional derivative:

$$
u(x, \tau)=u(x, 0)+u_{1}(x, 0)+u_{2}(x, 0)+\ldots
$$

We recover the approximate solution of the classical Black-Scholes equation when $\alpha=1$. In the context of the Mittag-Leffler fractional derivative, when we suppose $\alpha=1$, we get the following form:

$$
\begin{aligned}
u(x, \tau)= & u(x, 0)+u_{1}(x, 0)+u_{2}(x, 0)+\ldots \\
= & \max \left(e^{x}-1,0\right)+\left[k\left(\max \left(e^{x}, 0\right)-\max \left(e^{x}-1,0\right)\right)\right] \tau \\
& -\left[k^{2}\left(\max \left(e^{x}, 0\right)-\max \left(e^{x}-1,0\right)\right)\right] \tau^{2}+\ldots \\
= & \max \left(e^{x}-1,0\right)\left(1-e^{-k \tau}\right)+\max \left(e^{x}, 0\right) e^{-k \tau}
\end{aligned}
$$

Due to space limitation, all term are not written. Finally, we describe the analytical solution for the classical Black-Scholes equation (12) in the following form:
The method adopted here gives a solution which is in good agreement with the classical solution of the Black-Scholes equation (12). Let us prove our solution (45) is another representation for the traditional solution of the Black-Scholes equation using the following:

$$
V(S, E)=S \mathcal{N}\left(d_{1}\right)-E e^{-k \tau} \mathcal{N}\left(d_{2}\right),
$$

where $\mathcal{N}$ designs the normal distribution function, $d_{1}=\left(\log (S / E)+\left(r+\sigma^{2} / 2\right)(T-t)\right) / \sigma \sqrt{T-t}, \quad$ and $d_{2}=d_{1}-\sigma \sqrt{T-t}$. Note in the case "in the money," that is, $E<S$, we have high volatility; in other words, we have the following equation:

$$
\mathcal{N}\left(d_{1}\right)=\mathcal{N}\left(d_{2}\right)=1
$$
form:

From which, we rewrite equation (46) as the following

$$
V(S, E)=S-E e^{-k \tau}
$$

Equation (48) represents the value of the option "in the money." We recover this value with the solution proposed in 
equation (45) using the following reasoning. Note that in the case "in the money," there exists the following relationship:

$$
\begin{aligned}
\max \left(e^{x}, 0\right) & =e^{x}, \\
\max \left(e^{x}-1,0\right) & =e^{x}-1 .
\end{aligned}
$$

Now, we replace $e^{x}=S / E$ into equation (45), and using the assumption posed in equation (11), we obtain the following relationship:

$$
\begin{aligned}
u(x, \tau) & =\frac{S}{E}\left(1-e^{-k \tau}\right)-\left(\frac{S}{E}-1\right) e^{-k \tau} \\
& =\frac{S}{E}-e^{-k \tau}
\end{aligned}
$$
obtain

Multiplying the function $u$ by $E$ as in equation (11), we

$$
V(S, E)=S-E e^{-k \tau}
$$

We can see that the solution represented in equation (48) and the solution in equation (51) are the same. Thus, our analytical solution can be used in finance to determine the value of an option, satisfying the Black-Scholes equation.

Note that, in the money "call" is when the price of the underlying asset is higher than the strike price. It is in the interest of the holder of the option to exercise it. He/she has made good anticipations $(S>E)$; in this case, the value of the option is given by the formula $V=\max (S-E, 0)=S-E$. Out of the money "call" is when the price of the underlying asset is lower than the strike price. It is not in the interest of the option holder to exercise the option. $\mathrm{He} /$ she has made wrong expectations $(S<E)$; in this case, the value of the option is given by $V=\max (S-E, 0)=0$. In the money "put" is when the price of the underlying asset is lower than the strike price. It is in the interest of the option holder to exercise the option. He/she has made good anticipations $(S<E)$; in this case, the value of the option is given by $V=\max (E-S, 0)=E-S$. Out of the money "put" is when the price of the underlying asset is higher than the strike price. It is not in the interest of the option holder to exercise the option. He/she has made wrong anticipations $(S>E)$; in this case, the value of the option is given by $V=\max (E-S, 0)=0$.

\section{The Volatility of the Fractional Black-Scholes Equation}

In this section, we analyse the volatility of the fractional Black-Scholes equation using an analytical solution. In the market, we can buy the call and put with different strike prices and maturity. The volatility analyses the liquidity of the cost of the call and the put in the market. Many types of volatility can be generated by the Black-Scholes equation. In general, volatility measures risk in the financial markets. Volatility is used to control both upward and downward movements. It is calculated from log returns. The delta is part of the Greek letters of options. It is the derivative of the price of the call or put option in relation to the price of the underlying asset. It is used for trading, arbitrage, or hedging operations on options. It is an important indicator of market risk management. The Basel Committee on International Banking Regulation recommends that banks use delta for exposures to options. In reality delta is a sensitivity factor.

Here, we recall the formula of the volatility delta of the fractional Black-Scholes equation. The delta measures the sensitivity of the option price to a change in the price of the underlying security. Under variable changes, this is expressed as follows:

$$
\delta=\frac{\partial u}{\partial x}
$$

Using the approximate solution (52), it is clear that the sensitivity of the option price to a change in the price of the underlying security does not depend on the fractional-order and is given by

$$
\delta=\max \left(e^{x}, 0\right)
$$

Given the conditions in equation (11), the volatility of the option price to a change in the price of the underlying security is given by

$$
\delta=\max \left(\frac{S}{E}, 0\right)
$$

Earlier, we stated that the volatility studied in this paper does not depend on the order of the fractional derivative or the balance between the free interest rate and the volatility of the stocks $k$. This remark can be explained simply from the fact that, except for the first term $u(x, 0)$, we have the following relationship:

$$
\frac{\partial u_{i}}{\partial x}=0,
$$

for all $i=1,2,3, \ldots$.. Figure 1 shows the volatility surface of the option price to a change in the price of the underlying security in Figure 1.

The volatility surface gives the sensitivity of the option price when the asset price $S$ and the strike price of the underlying stock $E$ both vary in time. Let the asset price $S$ be fixed, and let the strike price of the underlying stock $E$ vary in time. The behavior of the volatility can be seen in Figure 2. In other words, we can see that when $S<E$, the volatility decreases rapidly and converges to zero. Let the underlying stock $E$ and the asset price $S$ vary in time. The behavior of the volatility can be seen in Figure 2. Thus, we can see in Figure 2 that when $E<S$, the volatility quickly increases linearly and converges to infinity. We note high volatility.

\section{Graphical Representations and Discussion}

In this section, we illustrate our results graphically. Specifically, we depict the behavior of the solutions of the fractional Black-Scholes equation obtained with numerical schemes and the recursive method. We begin with the approximate solution generated by the recursive method previously described in Section 7. In this context, we consider the solution with three iterations given by equation (43). Figure 3 shows the behavior of the approximate 


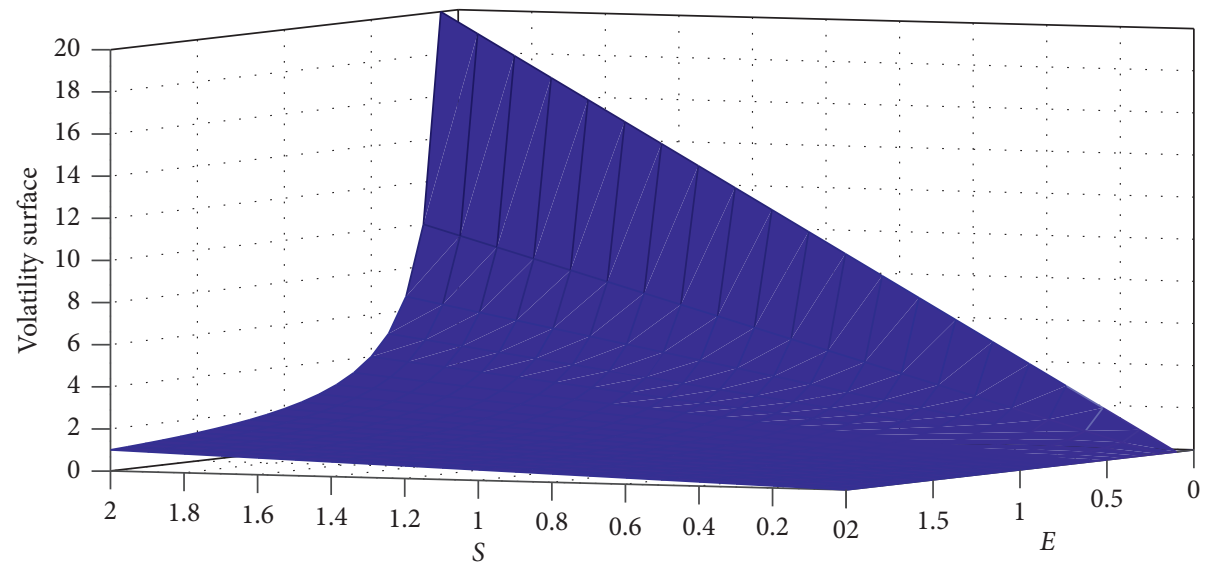

Figure 1: Volatility surface of the Black-Scholes equation.

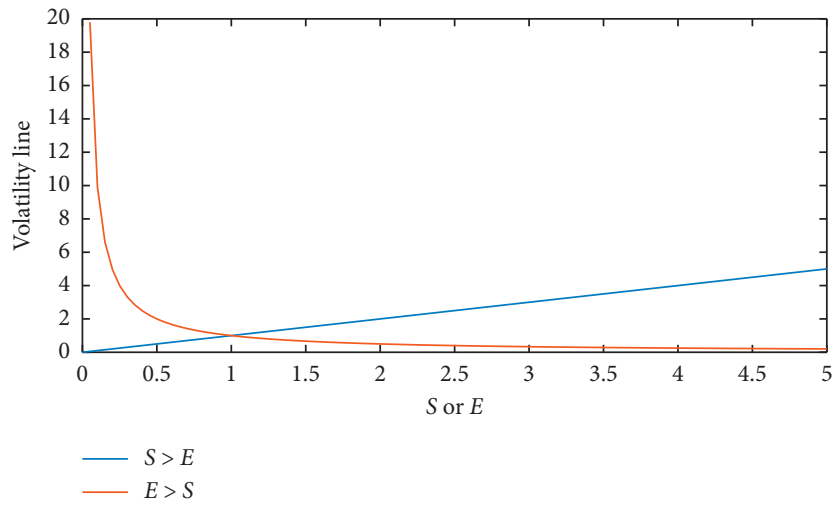

Figure 2: Volatility when $S<E$ and $E<S$.

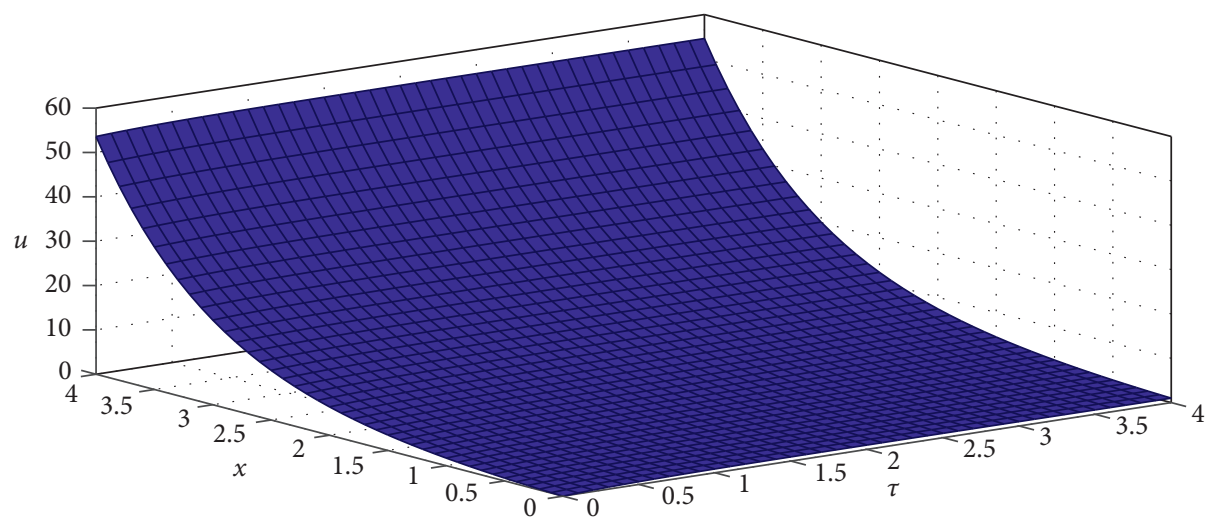

FIgURE 3: Behavior of the Black-Scholes equation with $\alpha=0.5$.

solution for the fractional Black-Scholes equation with the Atangana-Baleanu fractional derivative with order $\alpha=0.5$ and the balance between the free interest rate and the volatility of the stocks $k=2$. The constant $k$ is obtained with the risk-free interest rate to expiration $r=0.04$ and the volatility of the stocks $\sigma=0.2$ [35].

Figure 4 shows the behavior of the approximate solution for the fractional Black-Scholes equation with the Atangana-Baleanu fractional derivative with order $\alpha=1$ and the balance between the free interest rate and the volatility of the stocks $k=2$.
Figure 5 depicts the analytical solution with the recursive method and the approximate solution obtained with the Adams-Bashforth numerical scheme. We suppose $t=1$ and $\alpha=0.5$ and observed the numerical solution, and the approximate analytical solution is in good agreement.

The main question now is how to find the optimal order of $\alpha$. In our previous example, we depicted the figures by choosing the order $\alpha=0.5$ or $\alpha=1$. A complicated method is required to find the optimal order $\alpha$ in fractional calculus. First, we must collect the data in the considered market. 


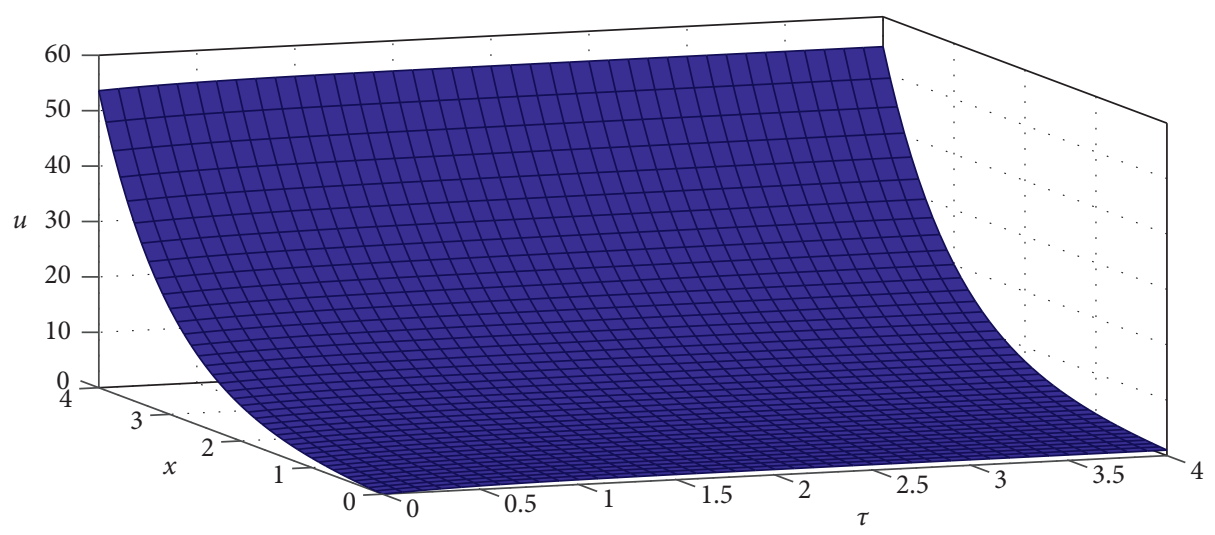

Figure 4: Behavior of the Black-Scholes equation with $\alpha=1$.

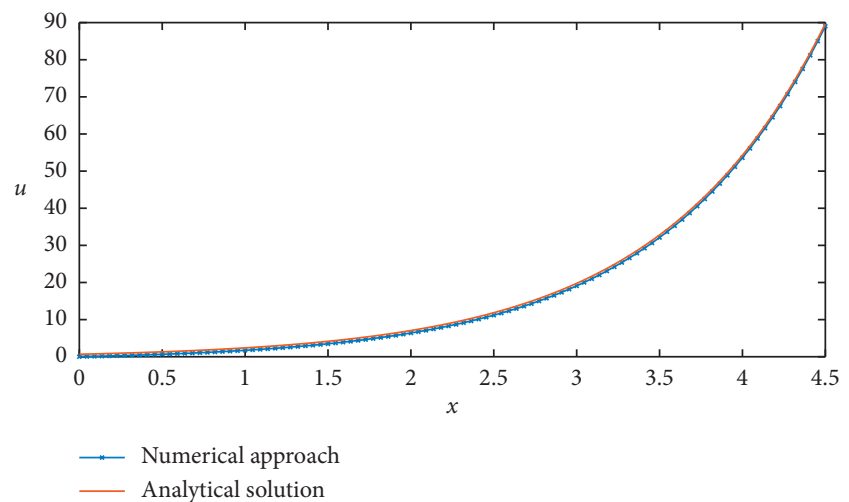

FIgURE 5: Analytical solution vs numerical approximation with $\alpha=0.5$.

TABle 2: Option values when $E<S$.

\begin{tabular}{lcccc}
\hline$x$ & $\tau$ & $u_{\text {Numerical }}$ & $u_{\text {Homotopy }}$ & $u_{\text {classic }}, \alpha=1$ \\
\hline 0.1541 & 0.01 & 0.3240 & 0.3242 & 1.1467 \\
0.4677 & 0.03 & 0.8455 & 0.8456 & 1.5381 \\
0.2401 & 0.05 & 0.5745 & 0.5747 & 1.1762 \\
0.1697 & 0.02 & 0.4924 & 0.4925 & 1.1457 \\
\hline
\end{tabular}

Second, we create a figure generated by the obtained data, and finally, we proceed by interpolation to find an order $\alpha$.

To support the numerical discretization, we describe in Table 2 the different values of the options generated by our numerical scheme, and we compare them with values obtained with the homotopy perturbation method. In other words, the robustness of the used numerical scheme is authenticated by comparing the numerical and analytical results in Table 2. In Table 2, we consider $\alpha=0.5$, the riskfree interest rate to expiration $\alpha=0.5$, the volatility of the stocks $\sigma=0.2$, the volatility of the stocks $k=2$ [35], and the strike price of the underlying stock $E=10$.

We mainly observe that the results in Table 2 are in good agreement with the results on the Black-Scholes equation studied in terms of the Caputo derivative in [35]. In general, we also notice that the order of the fractional derivative has a significant impact on the value of the options. In this investigation, the order $\alpha$ has a regulator impact, and we notice in Table 2 that the values obtained using the fractional order are more beneficial rather than the values obtained with the classical derivative in the financial market.

\section{Conclusion}

In this paper, we have discussed the numerical scheme and the analytical solution for the fractional Black-Scholes equation described by the Atangana-Baleanu derivative. As observed, the analytical solution of the fractional Black-Scholes equation with the Atangana-Baleanu fractional derivative is not trivial. We have used the Adams-Bashforth numerical scheme to approach the solution, as it is useful and straightforward for proposing the approximate solutions of the fractional Black-Scholes equation. We have also considered the liquidity of the cost of the call and the put in the market, namely, the volatility. The graphical representations have proved the good agreements between the analytical solution and the numerical solutions for the fractional Black-Scholes equation.

\section{Data Availability}

No data were used to support this study.

\section{Conflicts of Interest}

The authors declare that they have no conflicts of interest.

\section{References}

[1] M. H. Akrami and G. H. Erjaee, "Numerical solutions for fractional black-scholes option pricing equation," Global Analysis and Discrete Mathematics, vol. 1, pp. 9-14, 2015.

[2] F. Black and M. Scholes, "The pricing of options and corporate liabilities," Journal of Political Economy, vol. 81, no. 3, pp. 637-654, 1973.

[3] S. Kumar, D. Kumar, and J. Singh, "Numerical computation of fractional Black-Scholes equation arising in financial market," Egyptian Journal of Basic and Applied Sciences, vol. 1, no. 3-4, pp. 177-183, 2014.

[4] A. N. Fall, S. N. Ndiaye, and N. Sene, "Black-Scholes option pricing equations described by the Caputo generalized fractional derivative," Chaos, Solitons \& Fractals, vol. 125, pp. 108-118, 2019. 
[5] W. Chen, K. Du, and X. Qiu, "Analytic properties of american option prices under a modified Black-Scholes equation with spatial fractional derivative," Physica A: Statistical Mechanics and its Applications, vol. 491, pp. 37-44, 2017.

[6] L. Song and W. Wang, "Solution of the fractional blackscholes option pricing model by finite difference method," Abstract and Applied Analysis, vol. 2013, Article ID 194286, 10 pages, 2013.

[7] P. Sawangtong, K. Trachoo, W. Sawangtong, and B. Wiwattanapataphee, "The analytical solution for the blackscholes equation with two assets in the liouville-caputo fractional derivative sense," Mathematics, vol. 6, no. 8, p. 129, 2018.

[8] M. Yavuz and N. Özdemir, "A different approach to the European option pricing model with new fractional operator," Mathematical Modelling of Natural Phenomena, vol. 13, no. 1, p. 12, 2018.

[9] Z. Cen and A. Le, "A robust and accurate finite difference method for a generalized Black-Scholes equation," Journal of Computational and Applied Mathematics, vol. 235, no. 13, pp. 3728-3733, 2011.

[10] P. Phaochoo, A. Luadsong, and N. Aschariyaphotha, "The meshless local Petrov-Galerkin based on moving kriging interpolation for solving fractional Black-Scholes model," Journal of King Saud University-Science, vol. 28, no. 1, pp. 111-117, 2016.

[11] M. Yavuz and N. Özdemir, "European vanilla option pricing model of fractional order without singular kernel," Fractal and Fractional, vol. 2, no. 1, p. 3, 2018.

[12] M. Yavuz and N. Özdemir, "A quantitative approach to fractional option pricing problems with decomposition series," Konuralp Journal of Mathematics, vol. 6, no. 1, pp. 102-109, 2018.

[13] M. Yavuz, N. Özdemir, and Y. Y. Okur, "Generalized differential transform method for fractional partial differential equation from finance," in Proceedings of the International Conference on Fractional Differentiation and its Applications, pp. 778-785, Novi Sad, Serbia, 2016.

[14] A. Wakif, "A novel numerical procedure for simulating steady MHD convective flows of radiative casson fluids over a horizontal stretching sheet with irregular geometry under the combined influence of temperature-dependent viscosity and thermal conductivity," Mathematical Problems in Engineering, vol. 2020, Article ID 1675350, 20 pages, 2020.

[15] A. Wakif, A. Chamkha, T. Thumma, I. L. Animasaun, and R. Sehaqui, "Thermal radiation and surface roughness effects on the thermo-magneto-hydrodynamic stability of aluminacopper oxide hybrid nanofluids utilizing the generalized Buongiorno's nanofluid model," Journal of Thermal Analysis and Calorimetry, 2020, In press.

[16] A. Wakif, Z. Boulahia, S. R. Mishra, M. M. Rashidi, and R. Sehaqui, "Influence of a uniform transverse magnetic field on the thermo-hydrodynamic stability in water-based nanofluids with metallic nanoparticles using the generalized Buongiorno's mathematical model," The European Physical Journal Plus, vol. 133, p. 181, 2018.

[17] M. K. Nayak, A. Wakif, I. L. Animasaun, and M. S. H. Alaoui, "Numerical differential quadrature examination of steady mixed convection nanofluid flows over an isothermal thin needle conveying metallic and metallic oxide nanomaterials: a comparative investigation," Arabian Journal for Science and Engineering, 2020, In press.

[18] A. Zaib, U. Khan, A. Wakif, and M. Zaydan, "Numerical entropic analysis of mixed MHD convective flows from a nonisothermal vertical flat plate for radiative tangent hyperbolic blood biofuids conveying magnetite ferroparticles: dual similarity solutions," Arabian Journal for Science and Engineering, 2020, In press.

[19] A. Wakif, M. Qasim, M. I. Afridi, S. Saleem, and M. M. AlQarni, "Numerical examination of the entropic energy harvesting in a magnetohydrodynamic dissipative flow of Stokes' second problem: utilization of the gear-generalized diferential quadrature method," Journal of Non-Equilibrium Thermodynamics, vol. 44, no. 4, pp. 385-403, 2019.

[20] T. Thumma, A. Wakif, and I. L. Animasaun, "Generalized differential quadrature analysis of unsteady three-dimensional MHD radiating dissipative Casson fluid conveying tiny particles," Heat Transfer, vol. 49, no. 5, pp. 2595-2626, 2020.

[21] A. Atangana and D. Baleanu, "New fractional derivatives with nonlocal and non-singular kernel: theory and application to heat transfer model," Thermal Science, vol. 20, no. 2, pp. 763-769, 2016.

[22] S. Liao, "On the homotopy analysis method for nonlinear problems," Applied Mathematics and Computation, vol. 147, no. 2, pp. 499-513, 2004.

[23] K. M. Owolabi and A. Atangana, "Robustness of fractional difference schemes via the Caputo subdiffusion-reaction equations, chaos," Solitons \& Fractals, vol. 111, pp. 119-127, 2018.

[24] A. A. Kilbas, H. M. Srivastava, and J. J. Trujillo, Theory and Applications of Fractional Differential Equations, p. 204, North-Holland Mathematics Studies, Elsevier, Amsterdam, Netherlands, 2006.

[25] N. Sene, "Second-grade fluid model with Caputo-Liouville generalized fractional derivative," Chaos, Solitons \& Fractals, vol. 133, Article ID 109631, 2020.

[26] K. A. Abro and J. F. Gomez-Aguilar, "A comparison of heat and mass transfer on a Walter's-B fluid via Caputo-Fabrizio versus Atangana-Baleanu fractional derivatives using the Fox$\mathrm{H}$ function," The European Physical Journal Plus, vol. 134, no. 3, pp. 1-10, 2019.

[27] A. Atangana and T. Mekkaoui, "Trinition the complex number with two imaginary parts: fractal, chaos and fractional calculus," Chaos, Solitons \& Fractals, vol. 128, pp. 366-381, 2019.

[28] N. Sene, "Stokes' first problem for heated flat plate with Atangana-Baleanu fractional derivative," Chaos, Solitons \& Fractals, vol. 117, pp. 68-75, 2018.

[29] N. Sene, "SIR epidemic model with Mittag-Leffler fractional derivative," Chaos, Solitons \& Fractals, vol. 137, Article ID 109833, 2020.

[30] Q. Al-Mdallal, K. A. Abro, and I. Khan, "Analytical solutions of fractional walter's B fluid with applications," Complexity, vol. 2018, Article ID 8131329, 10 pages, 2018.

[31] T. Abdeljawad, M. A. Hajji, Q. Al-Mdallal, and F. Jarad, "Analysis of some generalized ABC-Fractional logistic models," Alexandria Engineering Journal, 2020, In press.

[32] M. Caputo and M. Fabrizio, "A new definition of fractional derivative without singular kernel," Progress in Fractional Differentiation and Applications, vol. 1, no. 2, pp. 1-15, 2015.

[33] T. Abdeljawad, R. Amin, K. Shah, Q. Al-Mdallal, and F. Jarad, "Efficient sustainable algorithm for numerical solutions of systems of fractional order differential equations by Haar wavelet collocation method," Alexandria Engineering Journal, 2020, In press.

[34] A. Atangana and K. M. Owolabi, "New numerical approach for fractional differential equations," Mathematical Modelling of Natural Phenomena, vol. 13, no. 1, p. 3, 2018.

[35] N. Özdemir and M. Yavuz, "Numerical solution of fractional black-scholes equation by using the multivariate padé approximation," Acta Physica Polonica A, vol. 132, pp. 10501053, 2017. 